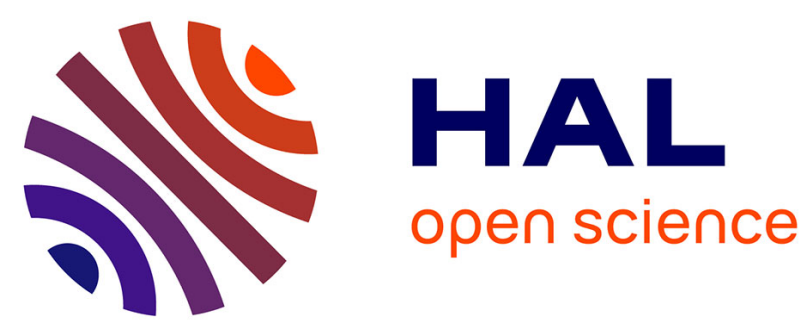

\title{
Circulating Mitochondrial DNA Level, a Noninvasive Biomarker for the Early Detection of Gastric Cancer
}

Julien Fernandes, Valérie Michel, Margarita Camorlinga-Ponce, Alejandro Gómez, Carmen Maldonado, Hilde de Reuse, Javier Torres, Eliette Touati

\section{- To cite this version:}

Julien Fernandes, Valérie Michel, Margarita Camorlinga-Ponce, Alejandro Gómez, Carmen Maldonado, et al.. Circulating Mitochondrial DNA Level, a Noninvasive Biomarker for the Early Detection of Gastric Cancer. Cancer Epidemiology, Biomarkers and Prevention, 2014, 23 (11), pp.2430-2438. 10.1158/1055-9965.EPI-14-0471 . hal-01652002

\section{HAL Id: hal-01652002 https://hal.science/hal-01652002}

Submitted on 29 Nov 2017

HAL is a multi-disciplinary open access archive for the deposit and dissemination of scientific research documents, whether they are published or not. The documents may come from teaching and research institutions in France or abroad, or from public or private research centers.
L'archive ouverte pluridisciplinaire HAL, est destinée au dépôt et à la diffusion de documents scientifiques de niveau recherche, publiés ou non, émanant des établissements d'enseignement et de recherche français ou étrangers, des laboratoires publics ou privés. 


\section{Circulating mitochondrial DNA level, a non-invasive biomarker for the early detection of gastric cancer}

Julien Fernandes ${ }^{1,2, a}$, Valérie Michel ${ }^{1,2}$, Margarita Camorlinga-Ponce ${ }^{3}$, Alejandro Gomez ${ }^{3}$, Carmen Maldonado ${ }^{4}$, Hilde De Reuse ${ }^{1,2}$, Javier Torres ${ }^{3}$ and Eliette Touati ${ }^{1,2^{*}}$

${ }^{1}$ Institut Pasteur, Department of Microbiology, Helicobacter Pathogenesis Unit, ${ }^{2}$ CNRS, ERL3526, 25-28 Rue du Dr. Roux, 75724 Paris cedex 15, France. ${ }^{3}$ Unidad de Investigacion en Enfermedades Infecciosas, UMAE Pediatria, IMSS, ${ }^{4}$ Hospital Infantil de Mexico, SS, Mexico.

${ }^{a}$ Present address: Unité U1152, Physiopathologie et Epidémiologie des Maladies Respiratoires, Université Paris Diderot, Faculté de Médecine, site Bichat, 16 Rue Henri Huchard, 75870 Paris cedex 18, France

Running Title: Circulating mitochondrial DNA as a gastric cancer biomarker

*Corresponding author: Dr. Eliette Touati, Institut Pasteur, Helicobacter Pathogenesis Unit, 25-28 rue du Docteur Roux, 75724 Paris cedex 15, France;

Email: etouati@pasteur.fr. Tel: +33140613785; Fax : +3314061364

\section{Financial support:}

Odyssey-Re Fundation and Fonds d'Aide à la Recherche, FAR project from the French National Society of Gastroenterology (SNFGE) to E. Touati,

CONACYT, Mexico to J. Torres (Grant 2007-C01-69450). J. Torres is a recipient of a exclusivity scholarship from Fundacion IMSS, Mexico.

Key words : Mitochondrial DNA, Biomarkers, Gastric cancer, Prevention/detection, Diagnostic test

\section{No Conflicts of Interest}

Words count: 4000

Total number of Figures: 3

Total number of Tables: 2 


\section{Abstract}

Background: Gastric cancer (GC) represents a major health burden worldwide often diagnosed at an advanced-stage. Biomarkers for screening and prevention of GC are missing. Changes in peripheral blood mitochondrial DNA (mtDNA) have emerged as potential preventive/diagnosis biomarker for cancer risk. We aimed to determine whether peripheral leucocytes mtDNA levels are associated with stages of the gastric carcinogenesis cascade.

Methods: We measured mtDNA by quantitative real-time PCR assay in peripheral leucocytes of 28 non-atrophic gastritis patients (NAG), 74 GC patients and 48 matched-asymptomatic controls. In parallel, serological level of IL-8 was determined.

Results: Mean mtDNA level was higher in GC patients ( $\mathrm{p}$-value $=0.0095$ ), compared to controls, with values $>8.46$ significantly associated with $\mathrm{GC}(\mathrm{OR}=3.93)$. Three ranges of mtDNA values were identified, interval I: $<2.0$, interval II: 2.0 to 20 and interval III: $>20$. Interval I included mainly NAG cases and few GC samples and interval III corresponded almost exclusively to GC patients. All controls felt in interval II, together with some NAG and GC cases; IL-8 levels were significantly higher in GC patients (p-value $<0.05$ ) with levels $>50 \mathrm{pg} / \mathrm{ml}$ observed exclusively in GC patients allowing to distinguish them within interval II. We validated mtDNA results in a second cohort of patients, confirming that mtDNA was significantly higher in GC than in patients with preneoplasia.

Conclusions: Circulating levels of mtDNA and IL-8 constitute a potential biomarker for the early detection of GC.

Impact: Our findings lead us to propose a new non-invasive method to detect patients with GC risk. 


\section{Introduction}

Gastric cancer (GC) represents a major health burden worldwide, affecting about onemillion people per year (1),(2). GC is often diagnosed at an advanced stage and consequently carries a poor prognosis (3). Importantly, if it is detected at an early asymptomatic stage, it can be curable (4); e.g. in Japan, a country with the highest incidence of GC, nation-wide strategies based on improved tests for detection of early GC or precancerous lesions have decreased the incidence of GC and increased survival rate (5).

Although GC arises from the complex interplay of environmental and host genetic factors $(6,7)$, the major risk factor is Helicobacter pylori infection, which is associated with more than $80 \%$ of all distal GC cases (8). The prevalence of $H$. pylori infection is high, with 80 $95 \%$ of the population infected in developing countries and up to $30 \%-40 \%$ of adults in industrialized countries (6). All infected individuals develop a gastritis which evolve to peptic ulcer diseases in about $10 \%$ of the cases, while gastric adenocarcinoma and mucosaassociated lymphoid tissue (MALT) lymphoma develop in $<3 \%$ and $0.3 \%$ of infected subjects, respectively $(9,10)$. Two types of GC can be distinguished, the intestinal and diffuse types. The intestinal type develops through progressive changes in the gastric mucosa from non-atrophic gastritis (NAG), atrophic gastritis, intestinal metaplasia (IM), dysplasia and gastric cancer (GC) (11). It has been shown that eradication of the infection at an early stage can reverse gastric lesions and more importantly, prevent the development of preneoplasia ((7),(12),(13)). A clinical study conducted in Japan demonstrated the efficacy of $H$. pylori eradication to reduce the incidence of GC (14), and confirmed that it was not enough to prevent all GC cases. These data raise the need for the development of biomarkers to detect precancerous lesions or early GC; the test should be a simple and non-invasive method, applicable in large scale screening programs. In the case of intestinal type GC, the measure of 
pepsinogens levels has been shown to be useful to detect gastric atrophy, although its utility in non-Asian countries is controversial (15). Thus, in spite of several efforts to develop biomarkers to identify patients at risk for distal GC (16), no efficacious screening test is yet available.

Mitochondria are essential organelles of eukaryotic cells that possess their own genome. Mitochondrial DNA (mtDNA) is a circular molecule present at 2-10 copies per organelle (17). Both, mutations and alterations of mtDNA content have been described in many different cancer types (18-20). MtDNA mutations have been detected at early stages of gastric carcinogenesis $(21,22)$. In $H$. pylori-infected patients, mtDNA mutations are significantly more frequent in GC patients than in cancer free patients (23). According to our previous studies, mtDNA mutations are induced in vitro in $H$. pylori-infected gastric epithelial cells and in the gastric mucosa of chronically infected mice (24),(25). A decrease in mtDNA content has also been described in most tumor tissues of advanced GC, compared with nearby non-tumor control tissue (26),(27),(28). Changes in peripheral blood mtDNA levels have recently emerged as a potential preventive/diagnosis biomarker associated with cancer risk (18). Circulating mtDNA levels were significantly higher in patients with urologic malignancies (29), breast (30) colorectal (31) and lung cancer (32, 33). These studies indicated that increased mtDNA content in peripheral blood is associated with elevated cancer risk. However, contrasting reports have found mtDNA depletion in the blood of patients with stage I breast tumors (34). A recent large prospective cohort study in women from Shanghai, found no association between the leukocyte mtDNA copy number and the presence of gastric tumor (35). However, they observed a positive association between a risk of developing GC and low mtDNA copy numbers in blood collected within the two years prior to cancer diagnosis. To our knowledge, there are no other reports on the variation of circulating mtDNA during the gastric carcinogenesis cascade, in particular comparing pre-neoplastic and 
neoplastic stages. To address this issue, in the present study we measured levels of mtDNA in the peripheral leucocytes of NAG, IM and GC patients. Our data showed significant variations in mtDNA levels during the progression from NAG to IM and to GC, supporting the notion that circulating mtDNA levels can be useful as potential biomarkers for the identification of early steps of gastric carcinogenesis.

\section{Material and methods}

\section{Study population}

Two cohorts of Mexican adult patients were studied. Cohort 1 included 48 healthy asymptomatic $H$. pylori negative blood-donors, $28 \mathrm{NAG}$ and $74 \mathrm{GC}$ patients for a total of 150 adults recruited during the period 2009-2011. The blood donors were recruited at the blood bank of the Instituto Mexicano del Seguro Social (IMSS), Medical Center SXXI in Mexico City. Cohort 2 was meant to validate results in cohort 1, and included 46 NAG patients, 31 patients with IM and 49 with GC for a total of 126 patients recruited during the period 19992002. Patients from both cohorts were adults who were attended for gastroduodenal diseases at IMSS. We selected patients who were not under treatment for cancer and who had not been treated with antibiotics, bismuth compounds, proton pump inhibitors and non-steroidal antiinflammatory drugs for at least two preceding weeks. Diagnosis was based on endoscopic examination and histopathology analysis (36). All patients and asymptomatic controls were informed and asked to sign a consent letter. The study was approved by the ethical committee from the National Council for Research on Health, IMSS.

\section{Collection of clinical samples and histological analysis}

For each patient, $10 \mathrm{ml}$ of blood were collected and gastric tissue specimens isolated. For patients with NAG or IM, gastric biopsies collected from both the antrum and the corpus were 
taken. For patients with GC, one fraction of tumoral and adjacent tissues were collected during surgery. Biopsies were immersed in formalin and processed for haematoxilin-eosin (H\&E) staining for histology analysis and diagnosis of gastric lesions. The presence of $H$. pylori was confirmed by Giemsa staining and serology.

\section{Circulating $m t D N A$}

Peripheral blood (10ml) was taken from each patient, and mononuclear cells were purified by centrifugation through a Ficoll-Hypaque density gradient. DNA was isolated from these cells using the salting-out micro-technique and frozen at $-70^{\circ} \mathrm{C}$ until tested for mtDNA quantification. The serum fraction was separated from cells and frozen at $-20^{\circ} \mathrm{C}$ until tested for serology to H. pylori antigens and IL-8 levels.

Determination of IgG against H. pylori antigens and of IL-8 in the serum fractions

H. pylori serology was determined with IgG antibodies against $H$. pylori whole-cell antigens and against CagA $H$. pylori protein using an enzyme-linked immune-absorbent assays (ELISA) previously validated by us (37). Serological levels of IL-8 were measured by ELISA (BD Biosciences).

\section{Quantification of mtDNA}

MtDNA levels were measured on DNA isolated from circulating leukocytes by quantitative Polymerase Chain Reaction (q-PCR) using the StepOne ${ }^{\mathrm{TM}}$ Plus Real-Time PCR system and FastStart Universal SYBR Green Master (Applied Biosystems) as previously described (38). MtDNA was quantified using a region in the $12 S$ ribosomal $R N A$ gene, and the nuclear encoded $18 S$ ribosomal $R N A$ gene as an endogenous reference. Primers used were: $12 S r R N A$ (forward): $\quad$ 5'-GCTCGCCAGAACACTACGAG; $\quad$ (reverse): CAGGGTTGGTGAAGATGGCG; $\quad$ 18SrRNA $\quad$ (forward): 
GAGAAACGGCTACCACATCC; (reverse): 5'-GCCTCGAAAGAGTCCTGTAT (39). The qPCR reaction was carried out in $20 \mu 1$ of total volume containing $5 \mu 1$ of DNA (200 pg), $10 \mu 1$ of FastStart Universal SYBR Green Master, $0.2 \mu \mathrm{l}$ of primers $(10 \mu \mathrm{M})$ using an initial denaturation step at $95^{\circ} \mathrm{C}$ for 10 minutes and 40 cycles of 15 secondes at $95^{\circ} \mathrm{C}$ and 1 minute at $60^{\circ} \mathrm{C}$. Samples were analyzed in triplicate. The average threshold cycle number values for nDNA $(18 S r R N A)$ and mtDNA $(12 S r R N A)$ were obtained. The relative mtDNA level was calculated using the delta $\mathrm{Ct}(\Delta \mathrm{Ct})$ of average $\mathrm{Ct}$ of nDNA and mtDNA $\left(\Delta \mathrm{Ct}=\mathrm{Ct}_{\mathrm{nDNA}}{ }^{-}\right.$ $\left.\mathrm{Ct}_{\mathrm{mtDNA}}\right)$ as $2^{\Delta \mathrm{Ct}}$ as previously described (40).

\section{Data analysis.}

The Student's t-test and Pearson's chi ${ }^{2}$ test were used to compare mtDNA level in peripheral blood between healthy subjects and patients at various stages of the gastric pathologies. Differences were considered significant for $\mathrm{p}$-values $<0.05$. For Odd Ratio (OR) determination the $95 \%$ confidence intervals were determined according to the Woolf's method (41). The sensitivity and specificity of mtDNA and IL-8 tests were calculated using receiver operator characteristic (ROC) analyses. Statistical tests performed using GraphPad Prism4 for Macintosh (GraphPad Inc., La Jolla, CA).

\section{Results}

\section{Characteristics of the studied cohorts}

The general characteristics of the patients included in this study are described in Table 1 . In Cohort $1,50 \%$ of the NAG patients were $H$. pylori-positive compared to $71 \%$ in the GC group. In the control group, we only selected $H$. pylori negative blood donors $(n=48)$. Patients in the NAG and GC groups were older (mean age 56 and 62 respectively), compared to individuals from the asymptomatic group (mean age 32) (p-value $<0.0001)$. Twenty-eight GC 
patients were diagnosed as diffuse-type, of which 6 had metastasis and 9 considered unresectable by surgeons because of a very advanced stage (late-stage). Twenty GC cases were of intestinal type, 7 presented hyperplasia, 7 metastasis and 1 case unresectable. In Cohort $2,78 \%$ of the NAG patients were $H$. pylori positive, compared to $84 \%$ in IM and $59 \%$ in GC. Overall, IM and GC patients were older compared to NAG patients.

\section{Levels of mtDNA in peripheral leukocytes from patients with $N A G$ and GC}

In Cohort 1, mtDNA was quantified in NAG and GC patients as described in material and methods, and compared with asymptomatic $H$. pylori negative subjects. In asymptomatic controls, the relative mtDNA values ranged from 2 to 17.09 , whereas in NAG group minimum and maximum mtDNA levels were 0.05 and 29.3 respectively. Even though in GC patients minimum mtDNA level was 1.74 , the maximum value measured was 60 (Figure 1A). Mean mtDNA levels were significantly higher in GC patients compared to asymptomatic controls $(2$-fold $)(p$-value $=0.0013)$ and to NAG patients $(2.2$-fold $)(p$-value $=0.0095)$. As reported in Figure 1A, three intervals of mtDNA values can be distinguished: whereas for all healthy individuals, mtDNA values grouped between 2 and 20 (interval II); in the mtDNA $<2.0$ group (interval I) only NAG (46\% of the cases) and GC (14\% of the cases) patients were observed. Importantly, samples with mtDNA $<0.5$ corresponded exclusively to NAG cases. In contrast, mtDNA values $>20$ (interval III) were not observed in healthy individuals, and only in $7 \%$ of $\mathrm{NAG}$, but in $28 \%$ of GC cases. We performed a ROC analyses (42) to determine a cut-off value of mtDNA that will differentiate GC cases from the asymptomatic H. pylorinegative controls and found a value of 8.46 , with a specificity of $80 \%$, but a sensitivity of $47 \%$. However, using this value we found that mtDNA $>8.46$ was significantly associated with GC $(\mathrm{OR}=3.93 ; 95 \% \mathrm{CI}=1.75-8.81)$ but not with NAG $(\mathrm{OR}=1.24 ; 95 \% \mathrm{CI}=0.42-3.67)$ (Table 
2), in agreement with an association between the presence of gastric malignancy and increased peripheral leukocytes mtDNA content.

An analysis of GC subgroups according to GC types, showed that the mean mtDNA values were similar among diffuse $(n=28)$ and intestinal types $(n=20)$ of GC patients, and significantly higher compared to healthy and NAG samples (Figure 1B). The severity of GC did not affect mtDNA as no significant differences were identified between cases with hyperplasia, metastasis or late-stage (unresectable) (Figure 1B). The presence of $H$. pylori infection had no effect on mtDNA content neither in NAG or in GC patients (Supplementary Figure S1). In addition, age had no influence on mtDNA levels among asymptomatic controls, NAG and GC groups, although a weak correlation was observed when considering the whole cohort (Supplementary Figure S2A). However, no effect of age was detected on mtDNA on both healthy and NAG samples, despite the presence of old individuals (Supplementary Figure S2A, insert graph Cohort 1). Furthermore, a slight mtDNA decrease in smokers compared to non-smokers was noticed (Supplementary Figure S3A).

In order to validate results in Cohort 1 , and to further test whether mtDNA levels differentiate patients with pre-neoplasia from NAG patients, mtDNA was quantified in Cohort 2 which included patients with NAG ( $\mathrm{n}=46)$, intestinal metaplasia (IM) $(n=31)$ and GC $(n=49)$ (Table 1). Samples from Cohort 2 were collected 10 years earlier (1999-2002) than Cohort 1 (20092011). This time lapse might be the reason why mtDNA levels in all patients of Cohort 2 were lower than in Cohort 1. Still, behavior between groups was similar and mean mtDNA levels were significantly higher in $\mathrm{GC}$ than in both NAG (p-value $=0.0012)$ and IM patients (pvalue $=0.0022)($ Supplementary Figure S4). Whereas no significant differences were observed between NAG and IM patients. In this cohort, higher mtDNA values $(>2.0)$ were significantly associated with GC, as compared with NAG (OR=8.48; 95\% CI 2.22-32.46) and with IM patients $(\mathrm{OR}=5.87 ; 95 \%$ CI 1.51-22.84). Thus, in agreement with data from Cohort 1 , 
peripheral leucocytes mtDNA levels shifted to higher values in GC patients as compared to pre-neoplastic NAG and IM patients. Age had no influence on mtDNA levels, in NAG, IM and GC groups, although a weak association was found for the whole cohort ( $\mathrm{p}$-value $=0.04$ ) (Supplementary Figure S2C). As for Cohort 1, the same analysis including only non cancer groups did not show any effect of age on mtDNA levels, despite the advanced age of some patients in these groups, specially among IM patients for which mean age was similar to GC patients (Supplementary Figure S2C, insert graph Cohort 2).

\section{Comparison of serological levels of IL-8 and mtDNA in NAG and GC patients}

Chronic gastric inflammation is associated with the promotion of carcinogenesis and inflammation can be correlated with plasma mtDNA levels (43). We wondered whether there was any correlation between plasma IL-8 and mtDNA in patients with NAG and GC using samples from Cohort 1. IL-8 levels are not affected by parameters as age (Supplementary Figure S2B). Compared to NAG patients and controls, IL-8 levels were significantly higher in GC patients (4- and 2.3-fold respectively) (p-value=0.016) (Figure 2A) either with diffuse (pvalue $=0.019)$ or intestinal type $(p$-value $=0.036)$ of cancer and with metastasis $(p$-value $=0.003)$ or unresectable ( $p$-value $=0.0015)$ (Figure 2B). However, no correlation was observed between IL-8 and mtDNA levels in GC patients. It should be noticed that among GC patients, IL-8 levels were higher in smokers than in non-smokers (Supplementary Figure S3B). Eighty percent of samples with IL-8 $>50 \mathrm{pg} / \mathrm{ml}$ corresponded to GC cases, among which $67 \%$ were in the mtDNA interval II. Moreover, $89 \%$ of GC samples with the highest mtDNA level (interval III), showed IL- $8<50 \mathrm{pg} / \mathrm{ml}$. For NAG patients, a weak but significant correlation was observed between IL- 8 and mtDNA $(\mathrm{r}=0.586 ; P=0.0041)$, although the analysis included only 25 samples. Thus, in most of the cases higher concentration of IL-8 differentiated GC 
patients with "normal" mtDNA levels (interval II), in spite of the lack of correlation between mtDNA and IL-8 levels (Figure 3).

\section{Discussion}

Gastric cancer remains the second cause of death by cancer, often detected at an advanced stage. Thus, there is a real need to develop appropriate screening strategies for its early detection. In Japan, one of the countries with the highest incidence of GC, strategies that include the eradication of $H$. pylori among young people, and periodic endoscopic examination have been proposed to reduce GC deaths (5). However, endoscopy is an invasive and costly method that requires special equipment and well-trained personnel to offer reliable diagnosis. Despite all recent developments in molecular diagnostic (16), identification of biomarkers with reliable predictive value are still unavailable. Peripheral blood mtDNA copy number has recently emerged as a potential preventive/diagnosis biomarker associated with the risk of various cancers (18),(19). Levels of circulating mtDNA were found significantly higher in patients with various type of tumors (29),(30),(31),(32),(44). Peripheral mtDNA content has also been proposed as a novel molecular marker for tracing tumor progression (34). In the present study, we found more variation of circulating leukocytes mtDNA level in patients with NAG or GC as compared with healthy subjects. We identify a distribution of samples according to three intervals of mtDNA levels measured as previously described (38),(40), intervals I: mtDNA<2.0; II with mtDNA comprised between 2.0 and 20.0 and III corresponding to mtDNA values $>20.0$. All asymptomatic individuals felt in the interval II, together with some NAG and GC patients. Importantly, in the interval I near half of NAG and very few GC patients but no healthy individuals were observed, whereas in the interval III almost only GC patients were found. Interestingly, low mtDNA values $(<0.5)$ corresponded only to NAG patients whereas high mtDNA levels $(>30)$ were exclusively observed in GC 
patients. According to these data, by a simple blood sampling, the classification of an individual in intervals I or III would be an indication of the presence of gastric inflammation and probable GC, respectively. This detection would constitute a first indication for the patients, upstream endoscopic investigation and specific follow-up.

In a recent large prospective study, no association between mtDNA copy number and GC was found (35). However a positive association between low mtDNA copy number and risk of GC was observed in blood collected within the two years prior to cancer diagnosis, suggesting that a decrease in mtDNA level is an early indicator of the malignant transformation of gastritis lesions. In agreement, in our study the lowest mtDNA levels correspond to NAG patients. Gastritis is characterized by a chronic inflammation of the gastric mucosa, associated with high production of oxidative species known to damage DNA, rendering mtDNA highly susceptible to damage and depletion. This is probably the reason for the lower mtDNA levels observed in some NAG cases. Although the role of mtDNA depletion in the cancer process remains unknown, it might decrease mitochondrial genes expression and impair the oxidative phosphorylation chain activity, leading to an increased production of ATP by glycolysis, a mechanism occurring during tumorigenesis, known as the Warburg effect (45). Low mtDNA copy number has also been reported associated to an increased risk in various cancers (46),(47). A lower blood mtDNA level has been described in stage I breast cancer as compared to advanced stages II to IV, leading to suggest that cancer cells deplete a larger amount of mtDNA in circulating cells at an early stage of cancer development. The initial decline in mtDNA content should be compensated at later stage of the cancer process to restore respiratory functions (34), which is in agreement with the increase we observed in many of the CG cases studied. This kinetic of mtDNA levels during the carcinogenic process would also be in agreement with results from Cohort 2 patients showing significant lower mtDNA levels in NAG and in precancerous IM patients, compared 
to most GC patients. This tendency was observed in Cohort 2 in spite of lower levels of mtDNA in all patients studied, which was probably due to the long time lapse of storage samples before testing. This observation would argue to the importance of using only fresh specimens to have reliable and clinically useful values. It also suggest that mtDNA levels cannot distinguish NAG patients and patients with early pre-neoplastic lesions.

Summarizing results from both cohorts, in healthy $H$. pylori negative individuals we observed a limited range of mtDNA levels (interval II) and values below this range were found mostly in patients with precancerous stages NAG and IM lesions (interval I); whereas values above this range were observed mostly in patients with GC (interval III). Based on these results and in agreement with a previous study (35), patients with decreased mtDNA levels potentially represent individuals with increased risk for GC, which should be monitored periodically to effectively prevent GC development. Whereas in patients with increased mtDNA, the presence of GC lesions should be suspected and further tested by endoscopy. However, in both, precancerous and GC groups we observed patients with mtDNA levels in the same range as healthy individuals. These patients need to be further studied to identify if they represent a group with a different carcinogenesis process or cancer stage. Among GC cases, we noticed a tendency, although not statistically significant, for mtDNA to increase according to the severity of the disease, from patients with hyperplasia or metastasis to laterstage (unresectable). In any case, it should be studied in a larger number of patients, to confirm if mtDNA may help differentiate GC stages and subtypes.

Chronic inflammation contributes to the promotion of cancer. Recently, a positive association has been reported between plasma level of pro-inflammatory cytokines, IL-6 and IL-8, and high GC incidence, suggesting them as potential biomarkers for GC (48). We did not find any correlation between IL-8 and mtDNA levels, indicating that the inflammatory process does not always drive the observed increased mtDNA levels in GC. In fact, we found 
mostly low IL-8 levels in GC cases with high mtDNA level (interval III). In addition, higher values of IL-8 were detected mostly in GC cases with "normal" mtDNA level (interval II), which supports that determination of both, mtDNA and IL-8, improves the identification of patients with GC in interval II. It is important to notice that most of these GC patients (mtDNA-interval II and IL-8 $>50 \mathrm{pg} / \mathrm{ml}$ ) were smokers. MtDNA levels can be also affected by other parameters as infections, that should be taken into account (49).

In conclusion, we identified three different ranges of peripheral leucocytes mtDNA levels, one present in all healthy H. pylori uninfected subjects and considered as "normal", one with lower mtDNA observed mainly in patients with gastric precancerous lesions and a third increased range corresponding almost exclusively to GC patients. According to our data, quantification of mtDNA by a simple blood sampling would permit an early detection of the presence of lesions and to design a personalized clinical follow-up of patients. Patients with low mtDNA level are likely to present precancerous or GC lesions and should be periodically monitored by endoscopy. Patients with high mtDNA level are more likely to already present GC and should be thoroughly studied by endoscopy. We should notice that there were patients with precancerous and with GC lesions with mtDNA levels in the same range as the asymptomatic group, which deserve more studies. Detection can be improved by testing in addition IL-8 levels, particularly in GC patients that present "normal" mtDNA (interval II). These data should be further validated in larger patient groups, also including patients from different geographical origin. Our findings indicate that testing for circulating mtDNA and IL8 might offer reliable minimaly invasive biomarkers, to screen populations at risk for GC. They pave the way to the development of circulating mtDNA measure as a predictive/earlydiagnostic biomarkers that are particularly needed in developing countries in Asia and Latin America, regions with the highest GC mortality rates. 


\section{References}

1. Parkin D M, Bray F, Ferlay J, Pisani P. Global cancer statistics, 2002. CA Cancer J Clin 2005; 55:74-108.

2. Ferlay J, Soerjomataram I, Ervik M, Dikshit R, Eser S, Mathers C, et al. GLOBOCAN 2012 v1.0, Cancer incidence and mortality worldwide: IARC CancerBase $\mathrm{N}^{\circ} 11$. Lyon France: International Agency for Research on Cancer. Available from http://globocan.iarc.fr. 2013.

3. Kamangar F, Dawsey S M., Blaser MJ, Perez-Perez GI, Pietinen P, Newschaffer CJ, et al. Opposing risks of gastric cardia and noncardia gastric adenocarcinomas associated with Helicobacter pylori seropositivity. J Natl Cancer Inst 2006;98: 1445-52.

4. Roukos DH. Early-stage gastric cancer: a highly treatable disease. Ann Surg Oncol 2003;11: 127-29.

5. Asaka M. A new approach for elimination of gastric cancer deaths in Japan. Int $\mathbf{J}$ Cancer 2013;132: 1272-76.

6. Resende C, Thiel A, Machado JC, Ristmäki A. Gastric cancer: Basic aspects. Helicobacter 2011;16: 38-44.

7. Bornschein J, Rokkas T, Selgrad M, Malfertheimer P. Gastric cancer: Clinical aspects, Epidemiology and Molecular Background. Helicobacter 2011;16: 45-52.

8. de Martel C, Ferlay J, Franceschi S, Vignat J, Bray F, Forman D, et al. Global burden of cancers attributable to infections in 2008: a review and synthetic analysis. Lancet Oncol 2012;13: 607-15.

9. Blaser MJ, Atherton JC. Helicobacter pylori persistence: biology and disease. J. Clin Invest 2004;113: 321-333.

10. Atherton JC. The pathogenesis of Helicobacter pylori-induced gastro-duodenal diseases. Ann Rev. Pathol. Mech. Dis 2006;1: 63-96.

11. Correa P. Human gastric carcinogenesis : a multistep and multifactorial process. First American Cancer Society Award Lecture on Cancer Epidemiology and Prevention. Cancer Re 1992;52: 6735-6740.

12. Zhou L, Sung JJ, Lin S. A five-year follow-up study on the pathological changes of gastric mucosa after H. pylori eradication. Chin Med J.2003;116: 11-14.

13. Ley C, Mohar A, Guarner J. Helicobacter pylori eradication and gastric preneoplastic conditions: a randomized, double-blind, placebo-controlled trial. Cancer Epidemiol Biomarkers 2004;13: 4-10.

14. Fukase K, Kato M, Kikuchi S, Inoue K, Uemura N, Okamoto S, et al. Effect of eradication of Helicobacter pylori on incidence of metachronous gastric carcinoma after endoscopic resection of early gastric cancer: an open-label, randomised controlled trial. Lancet 2008;372: 392-97.

15. di Mario F, and Cavallaro LG. Non-invasive tests in gastric diseases. Dig Liver Dis 2008;40: 523-30.

16. Cooke CL, Torres J, Solnick JV. Biomarkers of Helicobacter pylori-associated gastric cancer. Gut Microbes 2013;4:532-40.

17. Wallace DC. Mitochondrial DNA sequence variation in human evolution and disease. Proc Natl Acad Sci U S A 1994;91: 8739-46.

18. Meng S, Han, J. Mitochondrial DNA copy number alteration in human cancers. North Am $\mathrm{J}$ of Med Sci 2013;6: 22-25.

19. $\mathrm{Yu}$ M. Mitochondrial DNA copy number alterations in human cancers. eLS. John Wiley and Sons, Ltd: Chichester, 2012.

20. Carew JS, Huang P. Mitochondrial defects in cancer. Mol Cancer 2002; 1: 9. 
21. Kamalidehghan B, Houshmand M, Shafa Shariat Panahi M, Rezza Abbaszadegan M, Ismail $\mathrm{P}$, Bagher Shiroudi M. Tumoral cell $\mathrm{mtDNA}$ approximately $8.9 \mathrm{~kb}$ deletion is more common than other deletions in gastric cancer. Arch Med Res 2006;37: 848-53.

22. Maximo V, Soares P, Seruca R, Rocha AS, Castro P, Sobrinho-Simoes M. Microsatellite instability, mitochondrial DNA large deletions and mitochondrial DNA mutations in gastric carcinoma. Genes, Chromosomes and Cancer 2001;32: 136-43.

23. Hiyama T, Tanaka S, Shima H, Kose K, Kitadai Y, Ito M, et al. Somatic mutation of mitochondrial DNA in Helicobacter pylori-associated chronic gastritis in patients with and without gastric cancer. Int J Mol Med 2003;12: 169-74.

24. Machado A, Figuereido C, Touati E, Maximo V, Sousa S, Michel V, et al. Helicobacter pylori infection downregulates nuclear and mitochondrial DNA repair in gastric cells. Clin Cancer Res 2009; 15: 2995-3002.

25. Touati E. When bacteria become mutagenic and carcinogenic: lessons from $H$. pylori. Mutat Res 2010;703: 66-70.

26. Lee $\mathrm{HC}$, Yin $\mathrm{PH}$, Lin $\mathrm{JC}, \mathrm{Wu} \mathrm{CC}$, Chen $\mathrm{CY}, \mathrm{Wu} \mathrm{CW}$ et al. Mitochondrial genome instability and mtDNA depletion in human cancers. Ann N Y Acad Sci, 2005;1042: 10922.

27. Zhang G, Qu Y, Dang S, Yang Q, Shi B, Hou P. Variable copy number of mitochondrial DNA (mtDNA) predicts worse prognosis in advanced gastric cancer patients. Diagn Pathol 2013;8:173.

28. Wen SL, Zhang F, Feng S. Decreased copy number of mitochondrial DNA: A potential diagnostic criterion for gastric cancer. Oncol Lett 2013;6:1098-1102.

29. Ellinger J, Muller DC, Muller SC, Hauser S, Heukamp LC, von Ruecker A, et al. Circulating mitochondrial DNA in serum: a universal diagnostic biomarker for patients with urological malignancies. Urol. Oncol 2012;30:509-515.

30. Shen J, Platek M, Mahasneh A, Ambrosone CB, Zhao H. Mitochondrial copy number and risk of breast cancer: a pilot study. Mitochondrion 2010;10: 62-68.

31. Qu F, Liu X, Zhou F, Yang H, Bao G, He X, et al. Association between mitochondrial DNA content in leukocytes and colorectal cancer risk: a case-control analysis. Cancer 2011;117: 3148-55.

32. Bonner MR, Shen M, Liu CS, Divita M, He X, Lan Q. Mitochondrial DNA content and lung cancer risk in Xuan Wei, China. Lung Cancer 2009;63: 331-34.

33. Hosgood HD 3rd, Liu CS, Rothman N, Weinstein SJ, Bonner MR, Shen M et al. Mitochondrial DNA copy number and lung cancer risk in a prospective cohort study. Carcinogenesis 2010;31: 847-49.

34. Xia P, An H, Dang C, Radpour R, Kohler C, Fokas E, et al. Decreased mitochondrial DNA content in blood samples of patients with stage I breast cancer. BMC Cancer 2009;21: 454.

35. Liao LM, Baccarelli A, Shu XO, Gao YT, Ji BT, Yang GZ, et al. Mitochondrial DNA copy number and risk of gastric cancer: a report from the Shangai women's health study. Cancer Epidemiol Biomarkers Prev 2011;20:1944-9.

36. Camorlinga-Ponce M, Flores-Luna L, Lazcano-Ponce E, Herrero R, Bernal-Sahagun F, Abdo-Francis JM, et al. Age and severity of mucosal lesions influence the performance of serologic markers in Helicobacter pylori-associated gastroduodenal pathologies. Cancer Epidemiol Biomarkers Prev 2008;17:2498-504.

37. Camorlinga-Ponce M, Torres J, Perez-Perez G, Leal-Herrera Y, Gonzalez-Ortiz B, Madrazo de la Garza A, et al. Validation of a serologic test for the diagnosis of Helicobacter pylori infection and the immune response to urease and CagA in children. Am J Gastroenterol 1998;93:1264-70. 
38. Chatre L, Ricchetti M. Large heterogeneity of mitochondrial DNA transcription and initiation of replication exposed by single-cell imaging. J Cell Sci 2013;126:914-26.

39. Parone PA, Da Cruz. S, Tondera. D, Mattenberger. Y, James. DI, Maechler P, et al. Preventing mitchondrial fission impairs mitochondrial function and leads to loss of mitochondrial DNA. PLoS One 2008;3:e3257.

40. Fan AX, Radpour R, Haghighi MM, Kohler C, Xia P, Hahn S, et al. Mitochondrial DNA content in paired normal and cancerous breast tissue samples from patients with breast cancer. J Cancer Res Clin Oncol 2009;135:983-9.

41. Woolf B. On estimating the relation between blood group and disease. Ann Hum Genet 1955;19:251-3.

42. Delacour H, Servonnet A, Perrot A, Vigezzi JF, Ramirez JM. [ROC (receiver operating characteristics) curve: principles and application in biology]. Ann Biol Clin (Paris) 2005;63:145-54.

43. Cossarizza A, Pinti M, Nasi M, Gibellini L, Manzini S, Roat E, et al. Increased plasma levels of extracellular mitochondrial DNA during HIV infection: a new role for mitochondrial damage-associated molecular patterns during inflammation. Mitochondrion 2013;11:750-5.

44. Xu E, Sun W, Gu J, Chow WH, Ajani JA, Wu X. Association of mitochondrial DNA copy number in peripheral blood leukocytes with risk of esophageal adenocarcinoma. Carcinogenesis 2013.34: 2521-24.

45. Warburg O. On respiratory impairment in cancer cells. Science 1956;124:269-70.

46. Purdue MP, Hofmann JN, Colt JS, Hoxha M, Ruterbusch JJ, Davis FG, et al. A casecontrol study of peripheral blood mitochondrial DNA copy number and risk of renal cell carcinoma. PLoS One 2012; 7:e43149.

47. Xing J, Chen M, Wood C, Lin J, Spitz MR, Ma J, et al. Mitochondrial DNA content: Its genetic heritability and association with renal cell carcinoma. J Natl Cancer Inst 2008;100:1104-12.

48. Epplein M, Xiang YB, Cai Q, Peek RM, Jr., Li H, Correa P, et al. Circulating cytokines and gastric cancer risk. Cancer Causes Control 2013;24:2245-50.

49. Zhao S, Yang Y, Liu J, Liu H, Ge N, Yang H, et al. Association of mitochondrial DNA content in peripheral blood leukocyte with hepatitis B virus-related hepatocellular carcinoma in a Chinese Han population. Cancer Sci 2011;102: 1553-58. 


\section{Figure legends}

\section{Figure 1. Circulating mtDNA content in leukocytes of NAG and GC patients compared} to healthy blood donors in Cohort 1. A) MtDNA was quantified by quantitative real-time PCR (40), from DNA isolated from peripheral leukocytes isolated from healthy blood donors H. pylori-negative (open triangles), NAG (open circles) and GC (closed circles) patients. (40)Intervals I, II and III correspond to range of mtDNA level: $<2,2-20$ and $>20$, respectively. B) Peripheral leukocytes mtDNA level in NAG and GC patients according to the type of cancer either diffuse $(n=31)$ or intestinal $(n=20)$ (left part) or the presence of hyperplasia $(n=19)$, metastasis $(n=19)$ or late-stage cancer judged unresectable by surgeons $(n=17)$ (right part). As in control group, mean mtDNA levels are lower in NAG patients compared to GC of diffuse $(*)$ intestinal type $(* *)$ with hyperplasia $(*)$, metastasis $(* *)$ and late-stage cancer $(*)$. Each symbol corresponds to a single patient. Bars indicate mean \pm SEM. Samples were tested in triplicate in three independent experiments.

$*$ p-value $<0.05 ; * *<0.01: * * *<0.001$.

Figure 2. IL-8 serological levels in NAG and GC patients. A) IL-8 was measured by ELISA in sera from controls, NAG and GC patients from cohort 1. B) IL-8 levels were compared between diffuse and intestinal type of GC, as well as among GC with hyperplasia, metastasis or at late-stage. Bars correspond to mean \pm SEM. *p-value $<0.05 ; * *<0.01$

\section{Figure 3. Distribution of mtDNA levels according to IL-8 in healthy subjects, NAG and}

GC patients related to interval II. No correlation was observed in all cases between mtDNA and IL-8 levels. However, $75 \%$ of samples with IL-8>50pg/ml correspond to GC cases. Each symbol corresponds to a single patient. 
Table 1. Characteristics of the study population

\begin{tabular}{|c|c|c|c|c|c|}
\hline & & $\begin{array}{c}\text { Number } \\
\text { of } \\
\text { patients }\end{array}$ & $\begin{array}{l}\text { Mean age } \\
\text { (range) }\end{array}$ & $\begin{array}{c}\text { Sex ratio } \\
(\mathrm{M} / \mathrm{F})\end{array}$ & $\begin{array}{c}H . \text { pylori } \\
\text { positive } \\
\%\end{array}$ \\
\hline \multirow{4}{*}{ Cohort 1} & Healthy $^{\mathrm{a}}$ & 48 & $32(18-62)$ & 0.92 & - \\
\hline & $\begin{array}{l}\text { Non-atrophic gastritis } \\
\text { (NAG) }\end{array}$ & 28 & $\begin{array}{l}56(21-84) \\
\mathrm{p}<0.0001^{b}\end{array}$ & 2.25 & 50 \\
\hline & Gastric cancer (GC) & 74 & $\begin{array}{c}62(31-87) \\
\mathrm{p}<0.0001^{\mathrm{b}} \\
\mathrm{p}=0.019^{\mathrm{c}}\end{array}$ & 1.23 & 71 \\
\hline & $\begin{array}{l}\text { Total number of patients } \\
(\mathrm{NAG}+\mathrm{GC})\end{array}$ & \multicolumn{4}{|c|}{102} \\
\hline \multirow{4}{*}{ Cohort 2} & $\begin{array}{l}\text { Non-atrophic gastritis } \\
\text { (NAG) }\end{array}$ & 46 & $51(30-78)$ & 0.45 & 78 \\
\hline & $\begin{array}{l}\text { Intestinal Metaplasia } \\
\text { (IM) }\end{array}$ & 31 & $\begin{array}{r}60(33-80) \\
p=0.007^{d}\end{array}$ & 0.55 & 84 \\
\hline & Gastric cancer (GC) & 49 & $\begin{array}{r}62(31-86) \\
p=0.0003^{d}\end{array}$ & 1.88 & 59 \\
\hline & $\begin{array}{c}\text { Total number of patients } \\
\text { (NAG+IM+GC) }\end{array}$ & \multicolumn{4}{|c|}{126} \\
\hline
\end{tabular}

Two different groups of patients are analysed in the study. In each groups, samples were collected around the same period of time, from 2009-2011 for Cohort 1 and 1999-2002 for Cohort 2. ${ }^{\text {a }}$ Healthy subjects are H. pylori negative blood donors. P-values in the " Mean age » column represent statistical analyses for age distribution in, ${ }^{b} \mathrm{NAG}$ and GC patients compared to healthy individuals; ${ }^{\mathrm{c}} \mathrm{GC}$ patients compared to the NAG group (Cohort 1); ${ }^{\mathrm{d}} \mathrm{IM}$ and GC patients compared to the NAG group (Cohort 2). 
Table 2. Increased mtDNA is associated with significant risk for gastric cancer

\begin{tabular}{|c|c|c|c|c|c|}
\hline \multicolumn{6}{|c|}{ mtDNA* } \\
\hline Group & $<8.462$ & $>8.462$ & OR (CI 95\%) & $X^{2}$ & p-value \\
\hline Healthy & 40 & 8 & 1.00 & & \\
\hline NAG & 21 & 7 & $1.24(0.42-3.67)$ & 0.15 & 0.69 \\
\hline $\mathrm{GC}$ & 36 & 38 & $3.93(1.75-8.81)$ & 11.7 & 0.0006 \\
\hline
\end{tabular}

*cutoff value for mtDNA was $>8.462$ based on a ROC analyses 
A

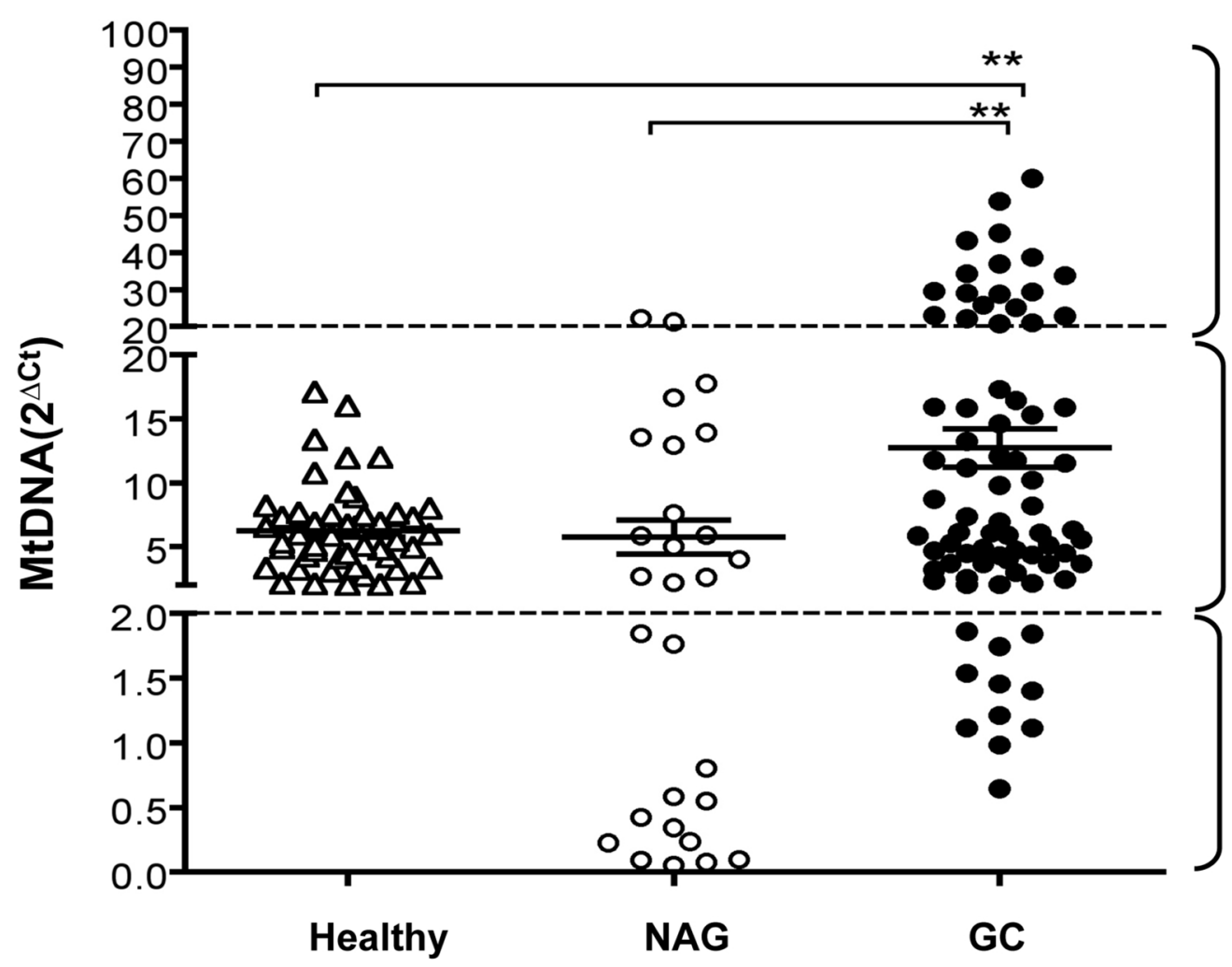

III

II

B

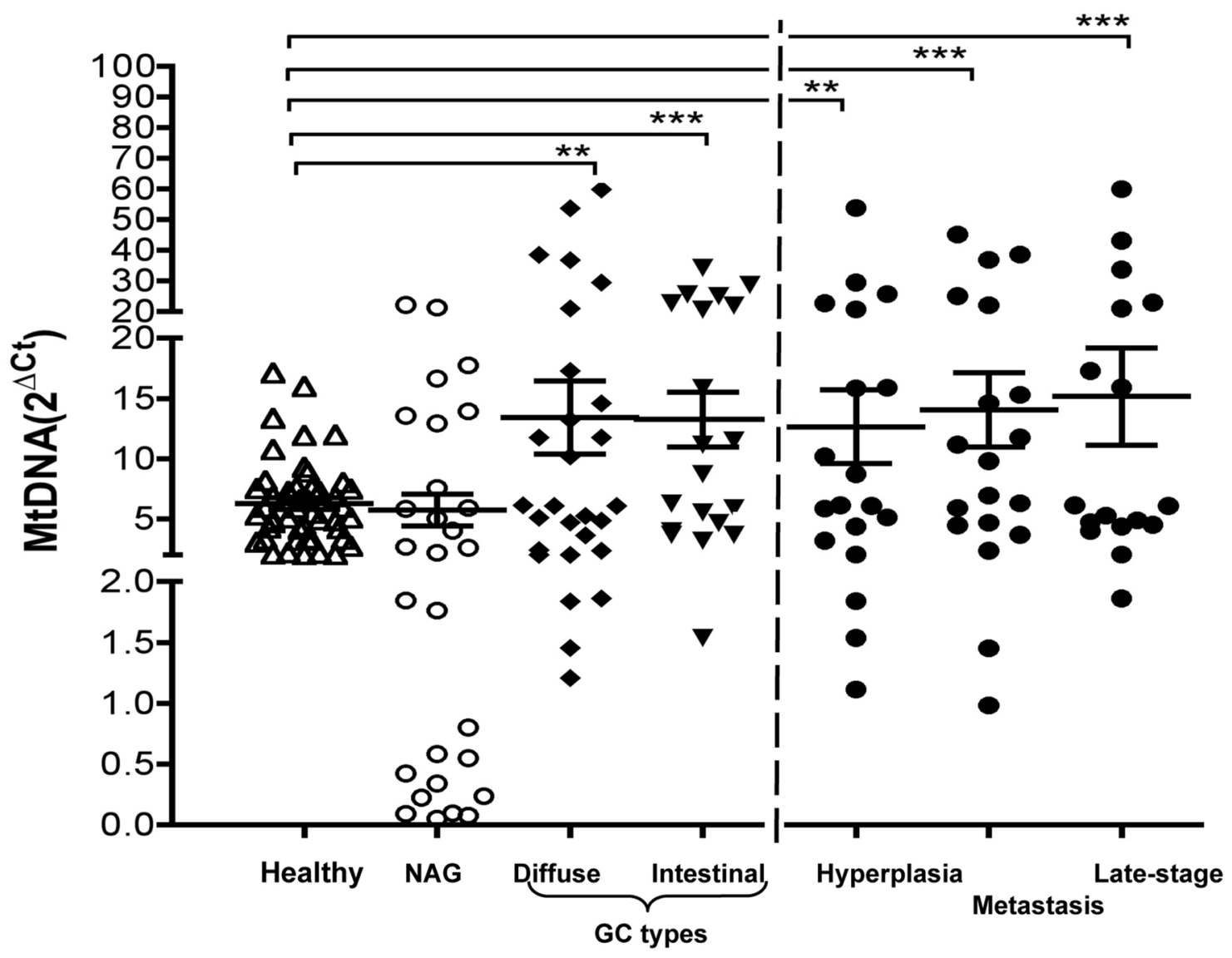

Figure 1 
A

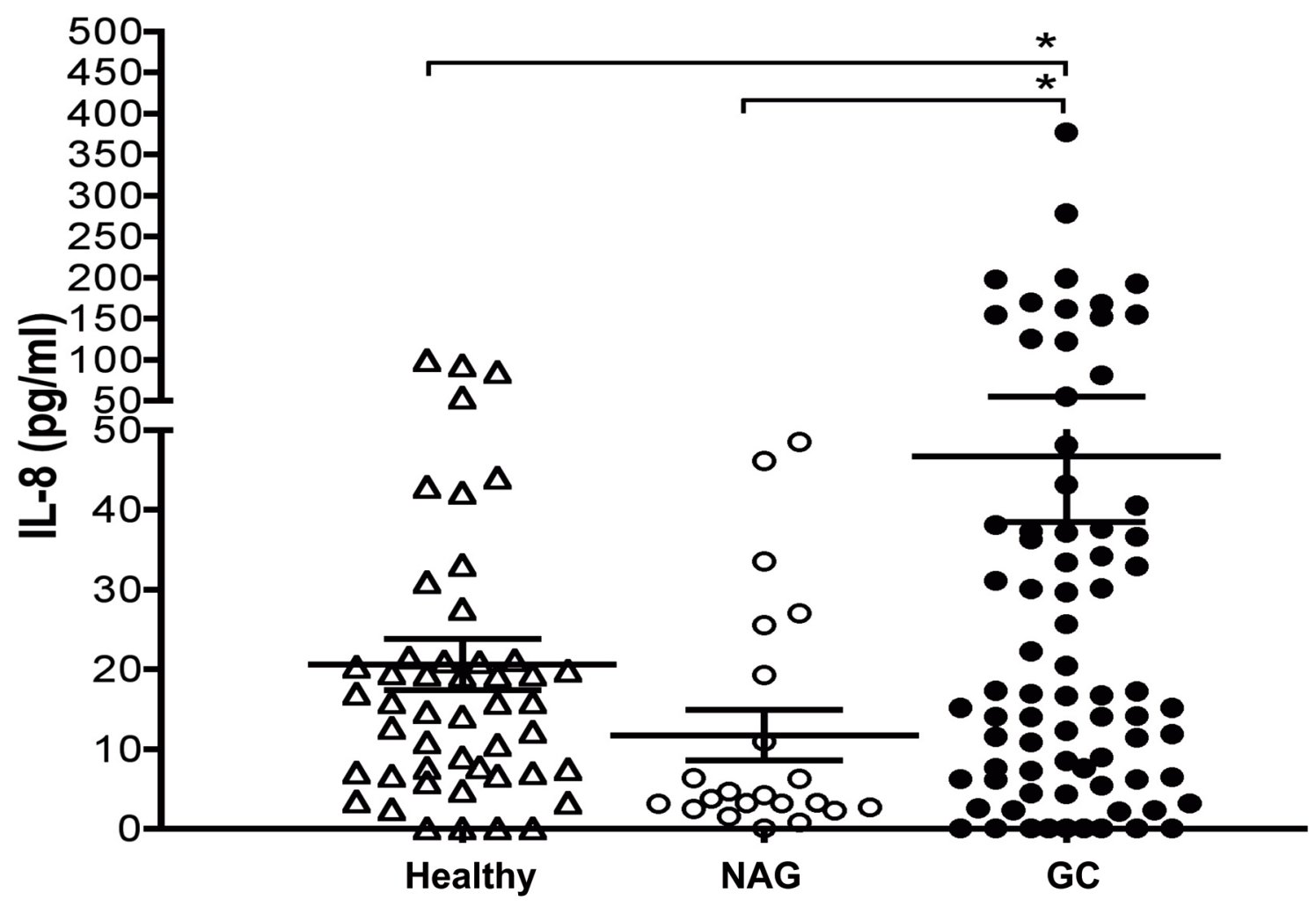

B

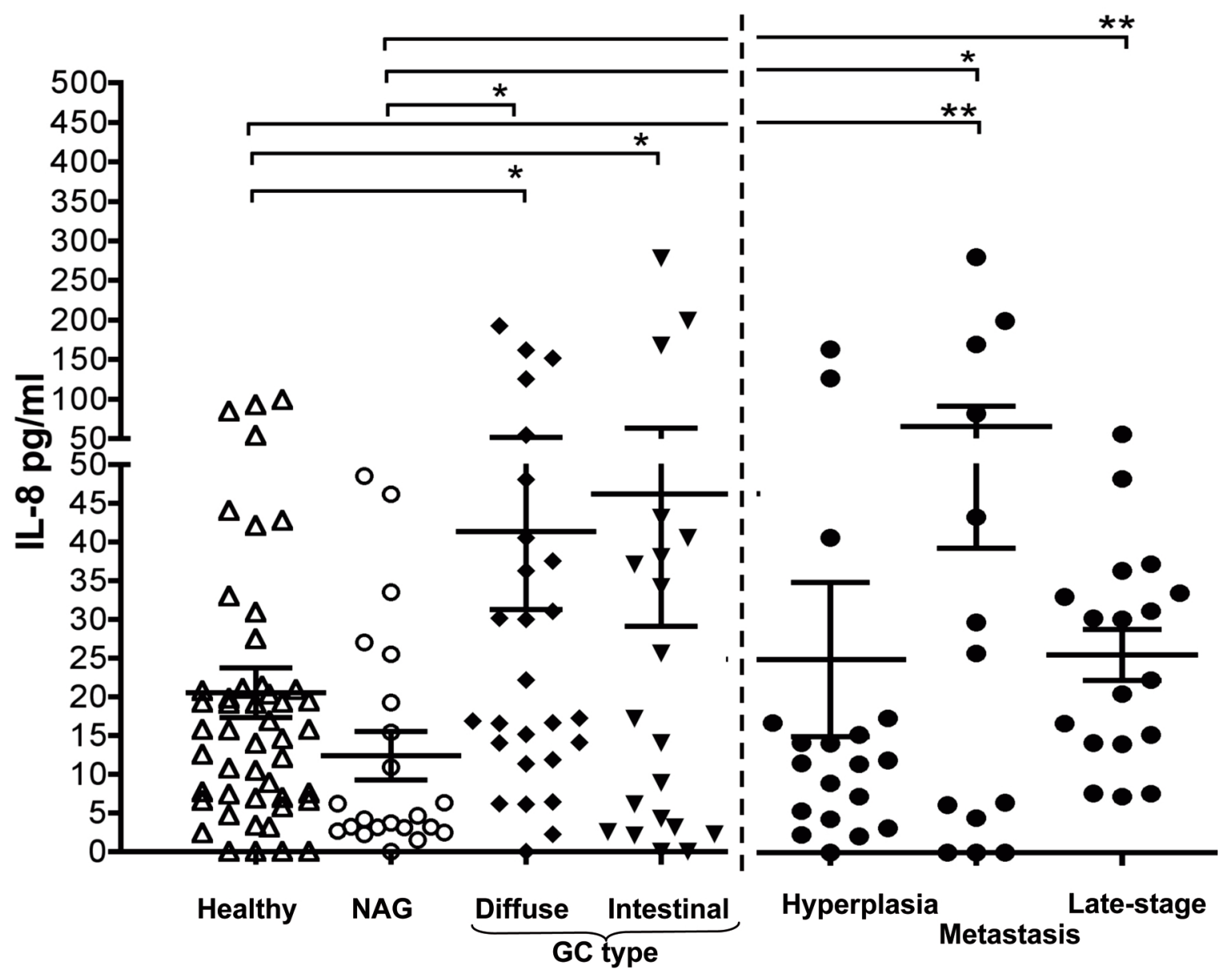

Figure 2 


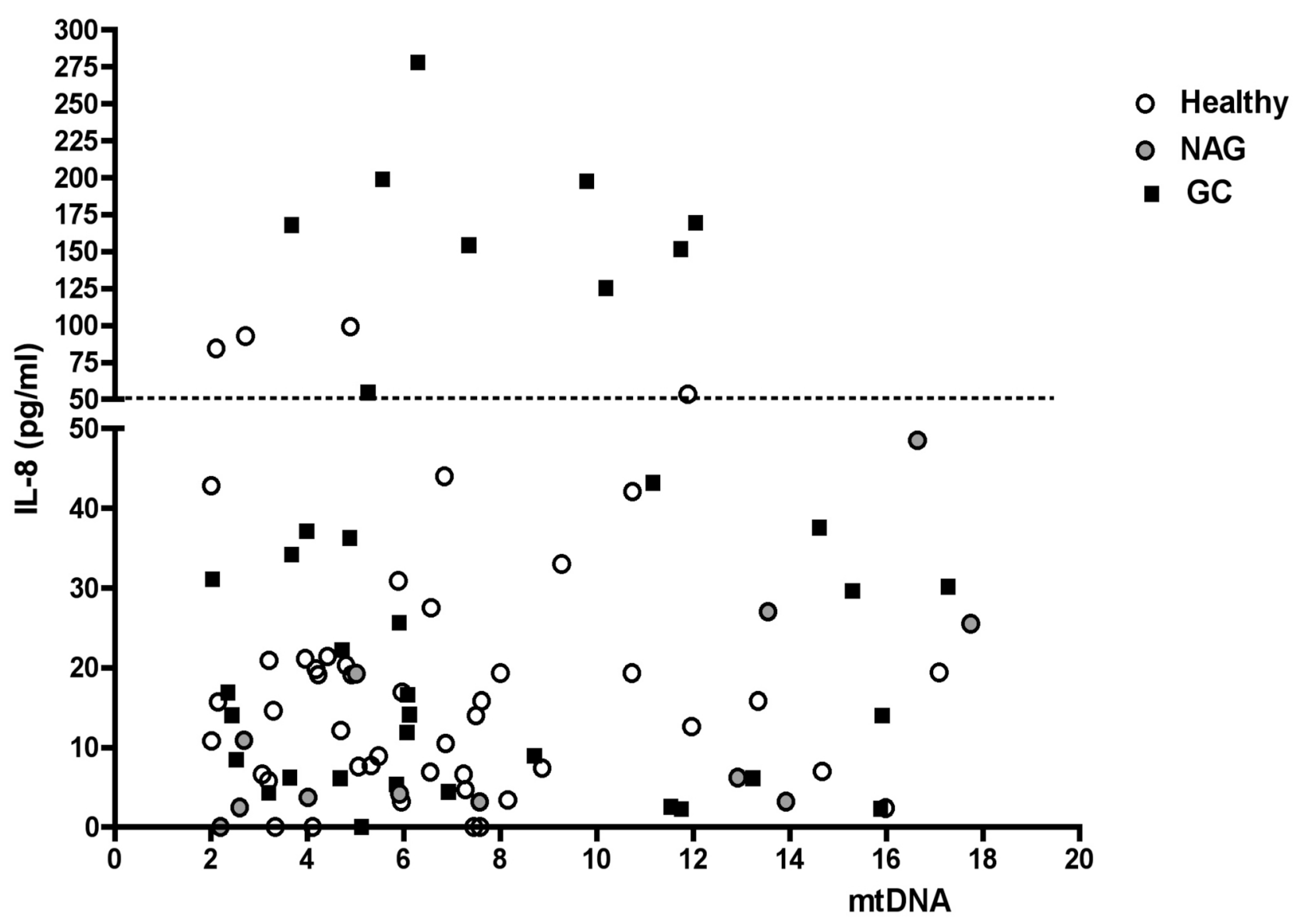

Figure 3 